\title{
Implications of the COVID-19 pandemic on orthopaedic surgical residency and fellowship training: lost opportunity or novel experience?
}

\author{
Morad Chughtai, Anas A. Minkara, Thomas E. Kuivila, Brendan M. Patterson \\ Department of Orthopaedic Surgery, Cleveland Clinic, Cleveland, OH, USA \\ Correspondence to: Anas A. Minkara, MD. Department of Orthopaedic Surgery, Cleveland Clinic, 9500 Euclid Avenue, Cleveland, OH 44103, USA. \\ Email: anas.minkara@gmail.com.
}

Submitted May 07, 2020. Accepted for publication Jun 16, 2020.

doi: $10.21037 /$ atm-20-3777

View this article at: http://dx.doi.org/10.21037/atm-20-3777

\section{Background}

The devastating COVID-19 pandemic has caused abrupt worldwide changes to healthcare infrastructure. The goals of these changes are to curb the pandemic, appropriately care for patients and prevent spread of this highly contagious pathogen (1). In the United States (US), the US Coronavirus Task Force has called on all hospital systems to delay or cancel nonessential elective procedures. The reasons for this are multiple, including but not limited to: (I) conserve personal protective equipment (PPE) such as gloves, gowns, eye shields, etc.; (II) decrease the risk of unnecessary iatrogenic transmission; (III) reallocate the health care work force to meet the overwhelming demands and challenges of increased patient volume and risk to healthcare providers that this pandemic brings; and (IV) free up beds and equipment such as ventilators, which effectively increases capacities and resources for care of COVID-19 patients (1).

From an orthopaedic surgical training perspective, the implications of these transitory changes on residency and fellowship training are very complex, dynamic, and overall, monumental. Our institution, which is a tertiary academic, multi-hospital enterprise with an orthopaedic surgery residency program and seven orthopaedic fellowship training programs has undergone daily changes to protocols, schedules, clinical rotations, and roles of residents, fellows, and staff since we began preparations in early March of 2020. In addition to residents, fellows in the specialties of Adult Reconstruction, Spine, Hand and Upper Extremity, Shoulder and Elbow, Foot and Ankle,
Pediatric Orthopaedics, as well as Sports Medicine have been redeployed to serve the shared purpose of preparing for the COVID pandemic in multiple capacities. Therefore, the purpose of this editorial is to discuss the potential implications of the COVID-19 outbreak on orthopaedic surgical residency and fellowship training.

\section{Systemic hospital changes pertaining to orthopaedic surgery}

In early March of 2020, all hospital personnel were notified that all non-essential business related travel would be cancelled to due COVID-19 to ensure employee and patient safety. At this point, the major implication to orthopaedic residency training would be the inability to travel to the 2020 American Academy of Orthopaedic Surgery (AAOS) conference, which would ultimately be cancelled anyway. In mid-March, didactic, academic, team sign out, as well as any group meetings were transitioned to a virtual platform. Shortly thereafter, residents were excused from out-patient clinic attendance in an effort to practice social distancing, diminish exam room and hallway congestion and thereby minimize exposure of both patients and healthcare workers. These initiative corresponded with the World Health Organization declaration that COVID-19 was a pandemic; with 37 cases confirmed in our home state at that time. One week later, it was announced coincident with Ohio Governor Michael DeWines' executive order that all nonessential elective cases would be cancelled. Of note, all recommendations of the Centers for Disease Control (CDC), US Coronavirus Task Force, as well as enterprise- 
level recommendations, such as hand hygiene, social distancing, and employee temperature checks, etc., were also implemented. It goes without saying, the enterprise and orthopaedic department have risen to the occasion of not only playing their role in the COVID-19 pandemic, but also meticulously meeting the specific ACGME requirement of orthopaedic surgery programs which states: "The program, in partnership with its Sponsoring Institution, must ensure bealthy and safe learning and working environments that promote resident well-being" (2).

\section{Magnitude of orthopaedic surgeries in health care systems}

Perhaps the most impactful change is the effect of cancelled or postponed elective orthopaedic procedures on training, as well as resident participation in clinic, indefinitely. The loss of surgical volume could be potentially significant in the grand scheme of training, not to mention hospital revenue, which is not a topic of discussion in this article. To put this into perspective, in the United States, musculoskeletal procedures are among the most commonly performed surgeries in any given hospital $(3,4)$. For example, in a study of the Healthcare Cost and Utilization Project database, which analyzed 8,000,000 surgical hospital stays, 7 of the 15 most common procedures involved the musculoskeletal system-these were knee arthroplasty $(700,100,9 \%)$, laminectomy $(468,200,6 \%)$, hip arthroplasty $(468,000$ stays, $6 \%)$, spinal fusion $(450,900,6 \%)$, and partial excision of bone $(338,000,4 \%)$. Two other common musculoskeletal procedures were trauma-related (treatment of hip fracture and treatment of other lower extremity fractures, which were $276,400,3 \%$ and $188,900,2 \%$, respectively) (4).

Furthermore, in a study of the 25 most frequent outpatient surgeries in 2015, muscle, tendon, and soft tissue procedures (mostly rotator cuff repair and trigger finger surgery) were the second most common type of surgery performed (6.6\% of 17.2 million ambulatory surgical visits), only second to cataract surgery. Incision or fusion of joint and destruction of joint lesion (mostly knee and shoulder arthroscopies) were ranked third, accounting for $5.3 \%$. Other musculoskeletal system procedures which ranked in the top 25 procedures were: excision of semilunar cartilage of knee (4.4\%), decompression of peripheral nerve (2.8\%), non-fracture, non-arthroplasty or procedures on the bone (2.4\%), partial excision bone (2.2\%), laminectomy, excision intervertebral disc $(2.2 \%)$ and bunionectomy or repair of toe deformities (1.6\%) (3).
In addition, a study of the of the 30 most common orthopaedic procedures in National Surgical Quality Improvement Program database from 2005-2011 of 101,862 patients, it appears that 21 of the 30 most common surgeries were mostly elective based on the judgment of the authors, which represented roughly $86 \%$ of all procedures (5). To emphasize, this is a speculation and extrapolation, and it is possible that procedures selected as elective by the authors of this study could have been done as emergency or essential cases (Table 1).

\section{Implications on residency and fellowship training}

As of March of this academic year (month 9 of 12 for the resident, and month 8 of 12 for the fellow academic year), it is safe to say that the typical resident or fellow has completed approximately $8(67 \%)$ or 7 months $(58 \%)$ of the planned training for their respective timelines. However, that leaves between $33 \%$ (for residents) to $42 \%$ (for fellows) of "non-routine or peri-pandemic" training remaining for the rest of that academic year.

In terms of the residency training year, using the data above this could extrapolate to a loss of roughly $28 \%$ of case volume for that residency year, and for fellows, a loss of roughly $36 \%$ of case volume for that fellowship year. However, the authors acknowledge that this is a very general and simple extrapolation, and it should be understood that different subspecialties may be more heavily impacted given the proportion of "elective procedures", such as Sports Medicine and Adult Reconstruction; whereas other subspecialties may have higher proportions of essential or emergent procedures such as Trauma or Orthopaedic Oncology. Furthermore, institutional variables such as location, trauma designation, academic versus private, and population also play a role in these numbers. In addition, it is unclear how long the COVID-19 Pandemic and associated protocol changes will last, with local estimates pointing to a peak of COVID activity occurring in midMay of 2020. If it were to last longer, then it could certainly extend into the next academic year for residents and have a substantially larger impact for residents. If it were to end earlier, then the impact would be less pronounced. Nevertheless, in a study of residency program case volume, the average number of cases was 1,900 (6), which is well above the 50th percentile of resident cases performed in $2019(n=1,500)$. However, for programs which offer limited rotations or exposure in a certain subspecialty, it is possible 
Table 1 Table of 30 most common orthopedic surgeries in order of their frequency (5)

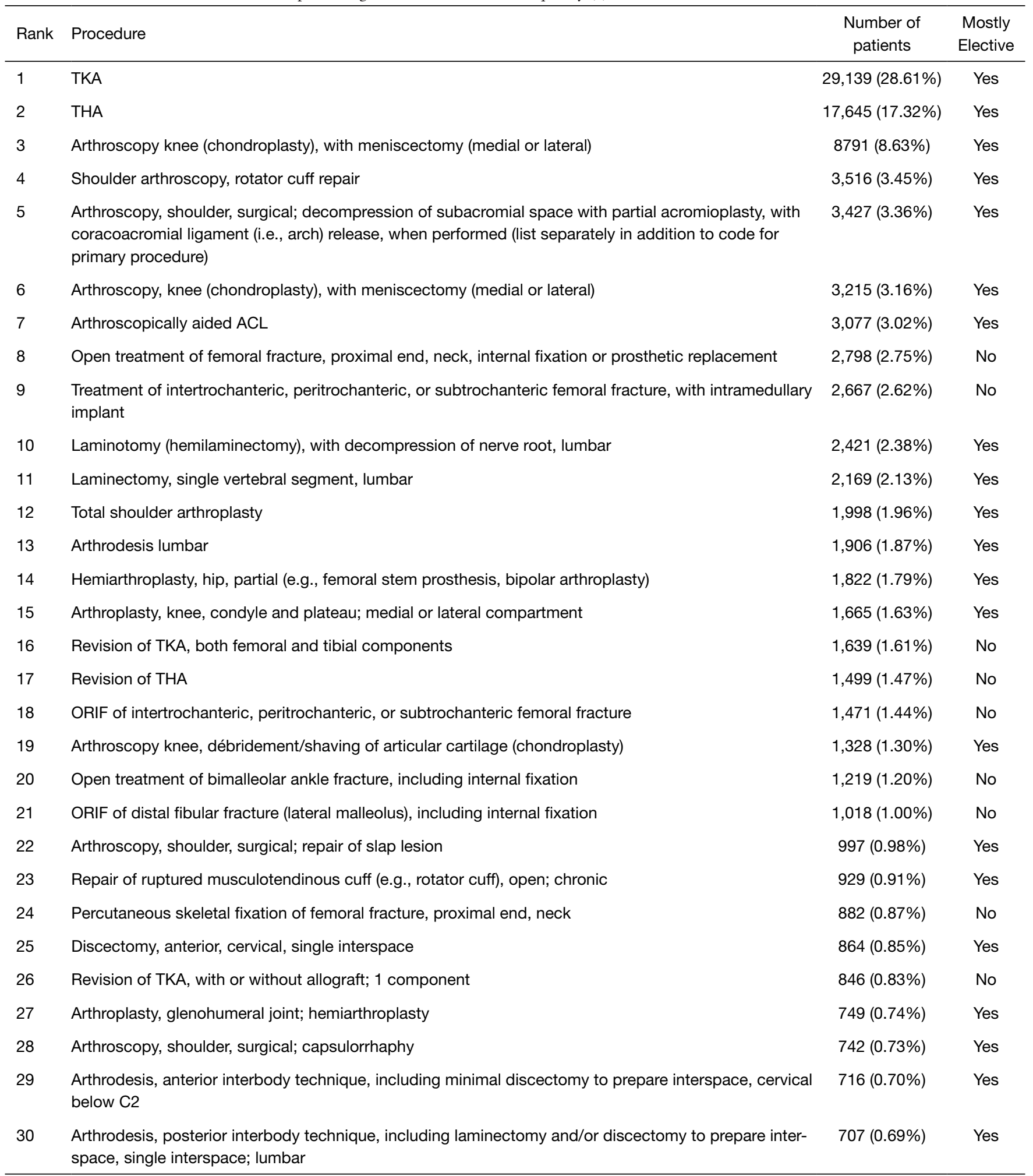


that they may not meet the requirement of being above the 10th percentile for a certain anatomical region, for which the residency program can be cited by the ACGME (7). Therefore, some programs may need to reconsider selective rearrangement of rotations for such residents in the future.

For fellows, the impact can be much more pronounced. Most orthopaedic fellowships are only 1-year in length, and the loss of volume of cases is likely more impactful. In this instance, fellowship with case-volume requirements may need to think ahead on potential plans to meet graduating criteria, whether it be extending the fellowship, or having a case-by-case evaluation of the competence and surgical skill of the individual fellow. Nevertheless, at this point in time, it is unknown when the protocols will be lifted as the situation remains very dynamic.

On the flipside of this, the impact of the massive infrastructure changes in healthcare and experience of the COVID-19 pandemic on orthopedic surgeons in training, although unfortunate, can indeed be valuable. Although, we are not the first-line providers for COVID-19 patients, there are many valuable lessons to be learned. For example, we have learned that by scaling back our practices, enormous amounts of resources are made available. Adopting methods such as rescheduling of clinical visits to virtual visits ( $80 \%$ at our institution at this time) may change the future of which types of visits can routinely be done through telemedicine, thereby increasing efficiency and improving safety.

At our institution, virtual academic lectures have been made available to residents in all subspecialties every day of the week. The utilized platforms have allowed residents to participate in educational lectures without interruption, offering them the opportunity to conduct group discussion via web conference. In addition to lectures, we have utilized this time to also conduct a series of leadership training seminars virtually, which has furthered resident education during this downtime.

From a clinical perspective, there has been re-purposing of closed ambulatory surgical centers (primarily for orthopedic elective procedures) to "Orthopaedic Acute Care Centers (OACC)", which serve as units for re-routing orthopaedic consults and emergencies away from our COVID-19 burdened hospitals and emergency departments to peripheral sites; which are staffed by restructuring the call schedules of orthopaedic residents and attendings. This helps ensure COVID-19 capacities are optimized for our designated hospitals and emergency departments. We have not only re-organized as an orthopedic department, but have coalesced with our enterprise to care for orthopaedic patients while minimizing exposures to COVID-19. It is more than likely that most generations of orthopedic trainees have not been a part of, experienced, and had to adapt to such a magnitude of a global health crisis. If there were to ever be a subsequent pandemic, the impact of the current one would certainly resonate, and the massive shift to our practices could be replicated, and hopefully enhanced.

Finally, although we conclude that there are implications of the COVID-19 pandemic on orthopaedic surgical residency and fellowship training; we ask: is this lost opportunity or a novel experience? We hope to open a forum for further discussion, other protocols and future research.

\section{Acknowledgments}

Funding: None.

\section{Footnote}

Provenance and Peer Review: This article was a free submission to the journal. The article did not undergo external peer review.

Conflicts of Interest: All authors have completed the ICMJE uniform disclosure form (available at http://dx.doi. org/10.21037/atm-20-3777). The authors have no conflicts of interest to declare.

Ethical Statement: The authors are accountable for all aspects of the work in ensuring that questions related to the accuracy or integrity of any part of the work are appropriately investigated and resolved.

Open Access Statement: This is an Open Access article distributed in accordance with the Creative Commons Attribution-NonCommercial-NoDerivs 4.0 International License (CC BY-NC-ND 4.0), which permits the noncommercial replication and distribution of the article with the strict proviso that no changes or edits are made and the original work is properly cited (including links to both the formal publication through the relevant DOI and the license). See: https://creativecommons.org/licenses/by-nc-nd/4.0/.

\section{References}

1. Coronavirus Disease 2019 (COVID-19) I CDC [Internet]. 
[cited 2020 Mar 23]. Available online: https://www.cdc. gov/coronavirus/2019-nCoV/

2. Acgme. ACGME Program Requirements for Graduate Medical Education in Orthopaedic Surgery. Available online: https://www.acgme.org/What-We-Do/ Accreditation/Common-Program-Requirements

3. Surgeries in Hospital-Based Ambulatory Surgery and Hospital Inpatient Settings, 2014 \#223 [Internet]. [cited 2020 Mar 23]. Available online: https://www.hcup-us. ahrq.gov/reports/statbriefs/sb223-Ambulatory-InpatientSurgeries-2014.jsp

4. Fingar KR, Stocks C, Weiss AJ, et al. Most Frequent Operating Room Procedures Performed in U.S. Hospitals, 2003-2012. In: Healthcare Cost and Utilization Project (HCUP) Statistical Briefs [Internet]. Rockville (MD):

Cite this article as: Chughtai M, Minkara AA, Kuivila TE, Patterson BM. Implications of the COVID-19 pandemic on orthopaedic surgical residency and fellowship training: lost opportunity or novel experience? Ann Transl Med 2020;8(18):1121. doi: 10.21037/atm-20-3777
Agency for Healthcare Research and Quality (US); 2006.

5. Molina CS, Thakore R V, Blumer A, et al. Use of the National Surgical Quality Improvement Program in orthopaedic surgery. Clin Orthop Relat Res 2015;473:1574-81.

6. Gil JA, Daniels AH, Weiss A-PC. Variability in Surgical Case Volume of Orthopaedic Surgery Residents: 2007 to 2013. J Am Acad Orthop Surg 2016;24:207-12.

7. Accreditation Council for Graduate Medical Education. Review Committee for Orthopaedic Surgery. Orthopaedic Surgery Minimum Numbers. Available online: https://www.acgme.org/Portals/0/PFAssets/ ProgramResources/260_ORS_Case_Log_Minimum_ Numbers.pdf 